\title{
A New Hybrid Chaotic Map and Its Application on Image Encryption and Hiding
}

\author{
Yang Cao \\ College of Telecommunications and Information Engineering, Nanjing University of Posts and Telecommunications, \\ Nanjing 210003, China \\ Correspondence should be addressed to Yang Cao; caoyeacy@njuptsast.org
}

Received 4 June 2013; Accepted 27 September 2013

Academic Editor: Dumitru Baleanu

Copyright ( 2013 Yang Cao. This is an open access article distributed under the Creative Commons Attribution License, which permits unrestricted use, distribution, and reproduction in any medium, provided the original work is properly cited.

A new hybrid chaotic map is constructed in this paper and applied to image encryption and hiding. MD5 is used as part of initial condition and control parameter to perturb the trajectory in order to further increase the security against plaintext-chosen attacks and differential attacks. Ciphered image is embedded into several carrier images to reduce suspiciousness and to increase robustness. The results demonstrate that the proposed scheme is of great security, robustness, and efficiency.

\section{Introduction}

Owing to the rapid development of the Internet, a lot of confidential information transfers across the world over public networks in all fields of the society. However, those networks are not suitable for the direct transmission of private messages $[1,2]$. Various types of files, such as text, image, and video [3-5] have to face the threat of unsafety. To keep secrecy and to make use of the networks already developed simultaneously, the actual and potential applications of cryptography extended prominently with the modern technological advance [6-8]. Since that, covert communication methods aroused the interest of many researchers and are becoming the world's attentive focus. Traditional encryption methods were an effective solution for information security $[6,9]$. Nonetheless, most of conventional ciphers, such as Data Encryption Standard (DES) [8], International Data Encryption Algorithm (IDEA) [10], and Advanced Encryption Standard (AES) [11], are not suitable for image encryption for three main reasons. The first reason is that the image size is almost always much greater than that of text, which needs much time to directly encrypt the image data using the software implementations of traditional cryptosystems. The second reason is laying on its sensitivity. The decrypted text must be exactly equal to the original text. However, this requirement is not necessary for image data. Because of human perception property and the high redundancy of image data, a decrypted image containing small distortion is usually acceptable. The third reason is because digital images' contents are strongly correlated. However, traditional cryptosystems never used those attributes, which hindered the promotion of encryption efficiency.

To adapt those unique characteristics of image data and to improve efficiency and security of image encryption, numerous special image encryption and hiding schemes were proposed. Among those schemes, the chaos based schemes have attracted the interest of many researchers from science and engineering realms. As ergodic, high sensitivity dependence on initial conditions, random-like behavior, continuous broad-band power spectrum, and unpredictability yet reproducible, chaos has huge potential applications in several vital fields of cryptosystem especially the image encryption. At the same time, different approaches for the transmission of information signals using chaotic dynamics were presented, such as chaotic masking, chaotic modulation, and chaotic switching. The idea of blending chaos theory into the cryptosystem came up since 1980s when Matthews [12] used chaos in applications to cryptology. After that numerous studies were done in the field of chaotic secure communication [13-16]. Baptista [17] proposed a text encryption using the ergodicity of the simple low-dimension and chaotic logistic map. But Álvarez et al. [18] pointed out that the method was not robust and secure enough. Afterwards, many improved encryption algorithms based on Baptista-type and other new ideas 
including the usage of fractional chaotic map were raised $[19,20]$. On the one hand, in order to guarantee the speed, some researchers continued to use one-dimensional chaotic maps for image encryption $[21,22]$. But analysis in $[23,24]$ showed that these schemes had some security defects due to some limitations such as small key space and weak security function in one-dimensional chaotic maps. Although chaos is infinite acyclic theoretically, in practical applications, there still exist many unpredicted problems, such as periodic degradation on account of finite precision limitation of computers [25] and low efficiency both caused by huge data and complicated chaotic behavior. The short cycle length problem makes many attacks feasible. On the other hand, some scholars adopt high-dimensional chaos systems into image encryption to enhance security for hyperchaos [26] having more complex dynamical characteristics. Although these methods like adopting high dimension maps can extend the cycle to some extent, the huge defect of computing time cost can totally offset its advantages.

In this paper, a novel image encryption and hiding scheme based on the new hybrid chaotic map will be proposed in order to keep the security and efficiency simultaneously. Primarily, a novel approach of constructing a new hybrid chaotic map will be given. Based on composition of three classic chaotic maps: logistic map, Hénon map, and Ikeda map, the new hybrid chaotic map demonstrates a more complex chaotic characteristic than most of single chaotic maps by Lyapunov exponent comparison, which means the hybrid map enjoys stronger encryption properties and is more sensitive dependence on initial conditions. The study results are of profound theoretical and practical significance because with the approach of composition, generating a brand new and stronger chaotic maps can be much simpler than before, which avoid the threat of known-system based attacks to a large extent. On account of the discrete map based properties, the cryptosystem also shows great performance on its efficiency. In the encryption scheme, the new hybrid chaotic map will be served as the key stream generator for encryption.

Meanwhile, MD5 values will be used as part of initial condition and control parameter to perturb the trajectory during iterations considering the capability to resist different attacks and the potential degradation from the computer limited precision problems. Generally, an encryption scheme is only highly connected to a fixed key which shows the weakness in plaintext-chosen and differential attack. In [27], the author's new idea that makes the initial key to be dependent on MD5 values of the plaintext made the encryption more sensitive and secure. In our scheme, MD5 values of plain image will be allowed to participate in much more place in the iteration of the new hybrid chaotic map, not only the initial keys but also the control parameters, which will further increase the sensitivity of plain image. Besides, with combination of MD5 values and fixed key, it is much more complex than the ordinary system [27]. Hence, it will guarantee the safety of this system since there are few works about the adaptive estimation of unknown secret parameters not to mention in the unknown chaotic map. According to the statistical tests involving key space, histogram analysis, information entropy, correlation coefficient, and mean square errors, this type of cryptosystem will be proved to be theoretically unbreakable by common attacks.

Furthermore, watermark technique and packet splitting strategy will be used together to embed ciphered image into several carrier images, which could make the ciphered image less conspicuous as well as more robust and meet the need of high efficient and robustness in covert packet networks. According to our simulations, those methods can greatly improve cipher's antinoise capability. Additionally, with MD5 embedding, an extra function of plain image confirmation can be added to the system based on its one-way associated property.

The rest of this paper will be organized as follows: Section 2 will give a descriptive presentation of the hybrid chaotic map and its great property. The design concept of proposed image encryption and hiding scheme will be described in Section 3. Simulation results and security analyses will be drawn in Sections 4 and 5. Finally, conclusions about the dissertation will be summarized.

\section{The New Hybrid Chaotic Map}

In this section, a new approach for constructing a new hybrid chaotic map will be put forward. In our scheme, the new hybrid chaotic map, which is constructed by composition of three classic chaotic maps: logistic map, Hénon map, and Ikeda map, reveals remarkable sensitivity to the initial condition and parameters comparing with their Lyapunov exponents. The detail process of construction and comparison results of Lyapunov exponents will be illustrated as follows.

2.1. Logistic Map. Logistic map [28, 29] is a polynomial mapping of degree 2 that exhibits chaotic behavior. The logistic map equation is given by

$$
x_{n+1}=\mu x_{n}\left(1-x_{n-1}\right) \text {, }
$$

where $x_{n} \in(0,1)$, control parameter $\mu \in(3.57,4]$. The bifurcation diagram of the map is shown in Figure 1. When the parameter approaches 4 , the chaotic systems exhibit a superb sensitivity to initial conditions.

2.2. Hénon Map. Hénon map was introduced by Hénon [4]. It is a simple two-dimensional reversible nonlinear chaotic map which iterates the point $\left(x_{n}, y_{n}\right)$ via the equation:

$$
x_{n+1}=1-a x_{n}^{2}+y_{n}, \quad y_{n+1}=b x_{n}
$$

where $a \in(0,1.4], b \in(0.2,0.314]$ are the control parameters on which the map depends. For $a=1.4, b=0.3$, the system can notably demonstrate chaotic behavior. Figure 2 shows the 10000 successive points obtained by iteration of the mapping. 


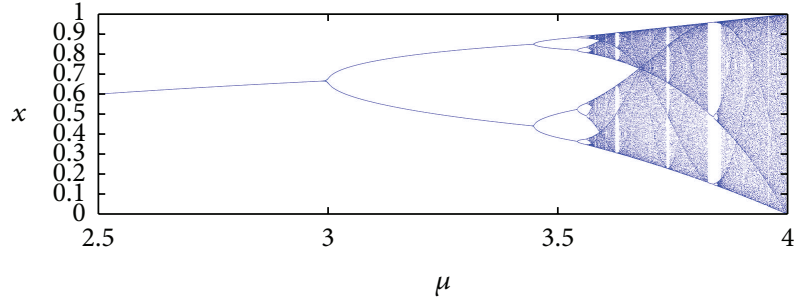

FIGURE 1: Bifurcation diagram of logistic map.

2.3. Ikeda Map. Ikeda map [30] is a discrete-time dynamical system. It is often used in a modified form, which is defined as the following equation:

$$
\begin{gathered}
x_{n+1}=1+u\left(x_{n} \cos t_{n}-y_{n} \sin t_{n}\right), \\
y_{n+1}=u\left(x_{n} \sin t_{n}-y_{n} \cos t_{n}\right),
\end{gathered}
$$

where $u$ is a parameter and $t_{n}=c-\left(6 /\left(1+x_{n}^{2}+y_{n}^{2}\right)\right)$.

For $c=0.4, u=0.9, x_{0}=y_{0}=0.1$, this system has a distinct chaotic attractor, which is shown in Figure 3.

2.4. The New Hybrid Chaotic Map. To generate a new compound mapping system, we composite (1)-(3) together with some necessary modification. The new hybrid mapping system is described as follows:

$$
\begin{gathered}
t_{n}=c-\frac{6}{1+x_{n}^{2}+y_{n}^{2}}, \\
w_{n+1}=1+u\left(w_{n} \cos t_{n}-s_{n} \sin t_{n}\right), \\
s_{n+1}=u\left(w_{n} \sin t_{n}-s_{n} \cos t_{n}\right), \\
x_{n+1}=1-a \cdot s_{n+1}^{2}+b \cdot w_{n+1}, \\
y_{n+1}=\mu \cdot w_{n+1}\left(1-s_{n+1}\right),
\end{gathered}
$$

where $w_{0}=s_{0} \in(0,1), c \in[0.1,0.4], u \in[0.8,0.85) \cup$ $(0.85,0.9], b / a>1, \mu>1$, and the phase diagram of hybrid map has demonstrated a notable chaotic attractor. For $w_{0}=$ $s_{0}=0.1, c=0.4, u=0.9, a=1.3, b=4, \mu=4$, the phase diagram of hybrid map is shown in Figure 4.

The value distribution over a random chosen initial key in $(0,1]$ is shown in Figure 5.

This construction approach is of profound theoretical and practical importance because with the idea of compositing, more and more new and sensitive chaotic maps are able to generate much simpler than before, which could vastly avoid the threat of known-system based attack.

2.5. Comparison of Lyapunov Exponent. In the preceding paragraphs, the descriptions of chaos phenomenon are qualitative rather than quantitative. To make a more quantitative analysis on those maps, their Lyapunov exponents will be calculated and compared in this section.

The Lyapunov exponent [31] defined as formula (5) is a common method to study the mean exponential rate of

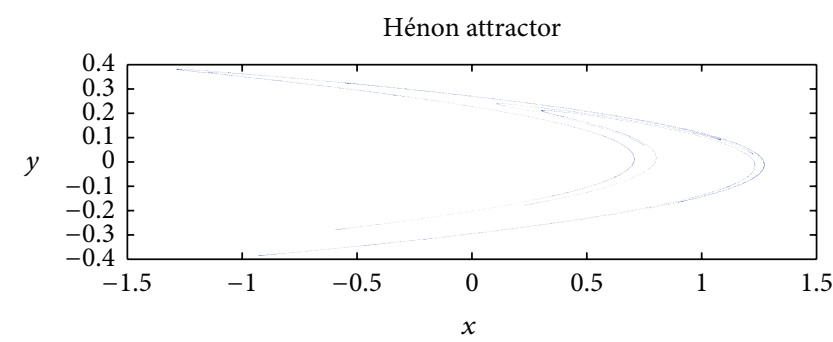

Figure 2: Phase diagram of Hénon map $\left(x_{0}=y_{0}=0.4, a=1.4, b=\right.$ $0.3)$.

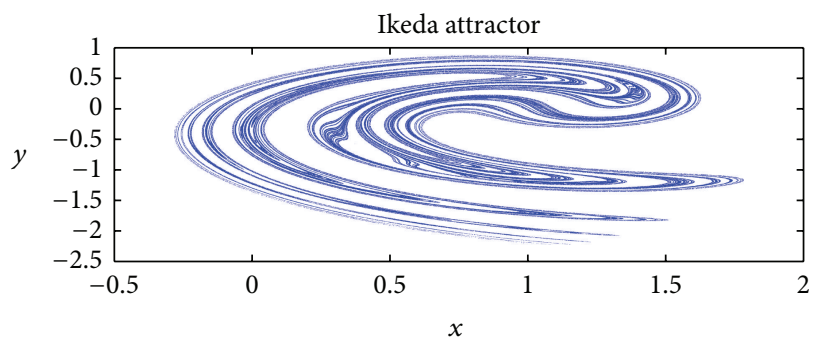

FIGURE 3: Phase diagram of Ikeda map $(u=0.9)$.

divergence of two initially close orbits over time, which indicates the sensitive dependence on initial conditions:

$$
L\left(f, x_{0}\right)=\lim _{n \rightarrow \infty} \frac{1}{n} \sum_{i=0}^{n-1} \ln \left|\left(f^{i}\right)^{\prime}\left(x_{0}\right)\right|,
$$

where $\left(f^{i}\right)^{\prime}$ is the derivative of the $i$ th iterate $\left(f^{i}\right)$.

$L<0$ : negative Lyapunov exponents are of dissipative or nonconservative systems, which indicates the orbit attracts to a stable fixed point or stable periodic orbit. Such systems exhibit asymptotic stability. The more negative the exponent, the greater the stability.

$L=0$ : a Lyapunov exponent of zero shows that the system is in some sort of steady state mode, which signs that the orbit is a fixed point (or an eventually fixed point).

$L>0$ : positive Lyapunov exponents reveal that the orbit is unstable and chaotic. Nearby points, no matter how close, will eventually diverge to any arbitrary separation [32].

Because sensitive dependence can be arisen only in some portions of a system (such as the logistic map), this separation is also a function of the location of the initial value and has the form $\left(f^{i}\right)^{\prime}\left(x_{0}\right)$ for an orbit of $f$ with starting point $x_{0}$. In a system with attracting fixed points or attracting periodic points, $L$ diminishes asymptotically with time. If a system is unstable, like pins balanced on their points, then the orbits diverge exponentially for a while but eventually settle down. For chaotic situation, the function will behave erratically [32].

For a 2D mapping system, there are two Lyapunov exponents $L_{x}, L_{y}$ for $x$ and $y$ orbits. Chaos only appears in the system which has at least one positive Lyapunov 


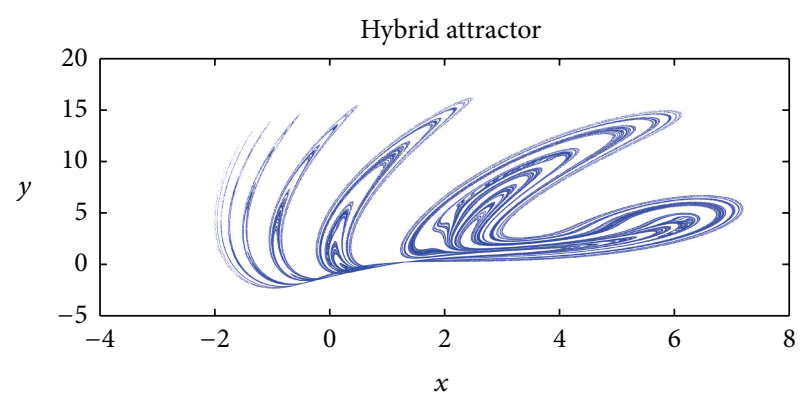

FIgURE 4: Phase diagram of the hybrid chaotic Map.

TABLE 1: Maximum Lyapunov exponent comparison.

\begin{tabular}{lcc}
\hline Maps & $L_{x}$ & $L_{y}$ \\
\hline Logistic map & 0.6913 & $\backslash$ \\
Hénon map & 0.4222 & -1.6261 \\
Ikeda map & 0.4926 & -0.7298 \\
Hybrid chaotic map & 0.5077 & -0.7329 \\
\hline
\end{tabular}

exponent. Figures 6,7, and 8 illustrate the Lyapunov exponent spectrums of the discussed maps.

Through the analysis of the Lyapunov exponent, the new hybrid chaotic map has one positive Lyapunov exponent, which can be theoretically convinced that the hybrid mapping system is in a chaotic state. Furthermore, through the comparison between the four mapping systems in Table 1 , it is easy to find out that the new hybrid system has the largest positive Lyapunov exponent along with the second largest negative Lyapunov exponent over all original two dimensional maps, which indicates that it has more obvious chaotic characteristics and is much more sensitive dependence on initial conditions than most of common single chaotic maps. Thus, it also denotes much stronger encryption abilities.

\section{Chaos-Based Image Processing Scheme}

3.1. Overall Process. In this section, the whole image processing scheme will be presented in detail, which will be constructed by two major parts-the encryption part and the hiding part. To enhance the security of image encryption, in the encryption part, a new algorithm based on the blending of the hybrid chaotic mapping system and MD5 hash will be proposed. In the hiding part, the Least Significant Bit (LSB) based watermarking embedded algorithm with packet division scheme will be adopted to hide and to split the ciphered image into different carrier images, which make the ciphered image less conspicuous as well as more robust and meet the need in high efficient packet communication.

The basic architecture of the whole chaos-based image processing scheme is displayed in Figure 9 while detailed explanation is presented afterwards.

\subsection{Encryption Scheme}

3.2.1. Generation of Initial Key. Our key includes initial user-set fixed real number key- $\mathrm{key}_{0}$, control parameter $a, b, c, u, \mu$ in (4), and MD5 values decided by the plain image. So the 128 bit MD5 value should be computed in advance, given by a color plain image $P$ with size of $M \times N \times 3$, where $P_{i, j \text {,dim }}$ represents pixel in $i$ th row $j$ th column in dimth color space (using RGB color space). After calculation, the 128 bit MD5 values of the plain image are split up into 32 4 bit blocks in decimal format-MD5(i) $1 \leq i \leq 32$. (To achieve a higher speed the block number can be reduced through an adjustable block splitting algorithm with size of plain image.) Then each MD5 block MD5(i) and the user-set key key ${ }_{0}$ will act on the initial values $w_{0}, s_{0}$ and the control parameter $\mu$ of the hybrid chaotic map which is given by the following equations for $i$ th iteration:

$$
\begin{gathered}
\operatorname{key}_{1}=\frac{\text { key }_{0}+1}{10^{{\text {length }\left(k e y_{0}\right)}_{1}},} \\
w_{0}=s_{0}=\frac{[\operatorname{MD} 5(i)+1]^{\mathrm{key}_{1}}}{\left[\operatorname{MD} 5(i)_{\max }+1\right]^{\mathrm{key}_{1}}}, \\
\mu=4-\frac{w_{0}}{10},
\end{gathered}
$$

where $\operatorname{MD} 5(i)_{\max }=16$, for splitting up to 32 parts.

Hence, the key not only disturbs the initial value of the new hybrid chaotic map but also adds perturbations to the control parameter to the chaos trajectory, which signifies that each pseudorandom sequence is generated from switching the trajectory from different hybrid chaotic map. Meanwhile, with the on-way property of MD5 hash, the decrypted image is able to audit with MD5. Since there is seldom work about the adaptive estimation of the unknown secret parameters in the unknown chaotic mapping systems even if the cryptanalyst obtains the whole trajectories, it not only guarantees the safety but also makes verification available.

3.2.2. Encryption Algorithm. As previously discussed, for $i$ th iteration, the initial condition $w_{0}, s_{0}, \mu$ is given by (6) and the basic idea of the algorithm is shown in Figure 9. The detailed encryption flow is reflected in the next several steps. After that, the ciphered image will be created.

Step 1 (generation of key stream). Subiterative calculation of the chaotic sequence $X, Y$ by (4) as the algorithm described below. The time of subiterations is equal to $M \times N-1$. Then a chaotic key stream is generated. Consider

$$
\begin{aligned}
& \text { for } n=0: 1: M \times N-1 \\
& \begin{array}{l}
t=c-\frac{6}{1+w^{2}(n)+s^{2}(n)}, \\
w(n+1)=1+u[w(n) \cos t-s(n) \sin t], \\
s(n+1)=u[w(n) \sin t-s(n) \cos t], \\
x(n+1)_{n+1}=1-a \times s^{2}(n+1)+b \times w(n+1), \\
y(n+1)_{n+1}=\mu \times w(n+1) \times[1-s(n+1)],
\end{array}
\end{aligned}
$$

end. 


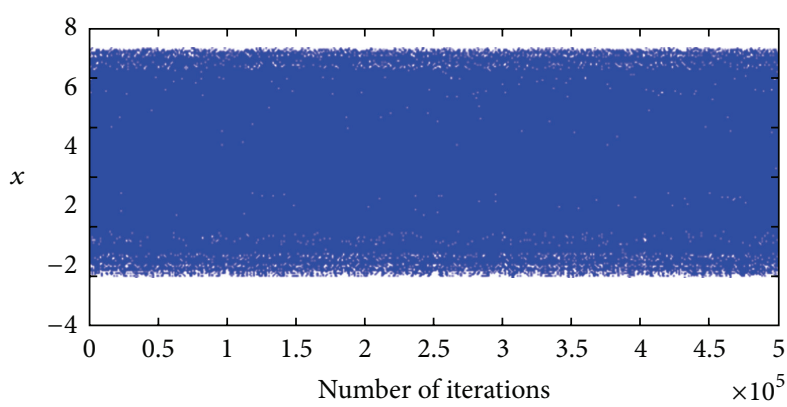

(a)

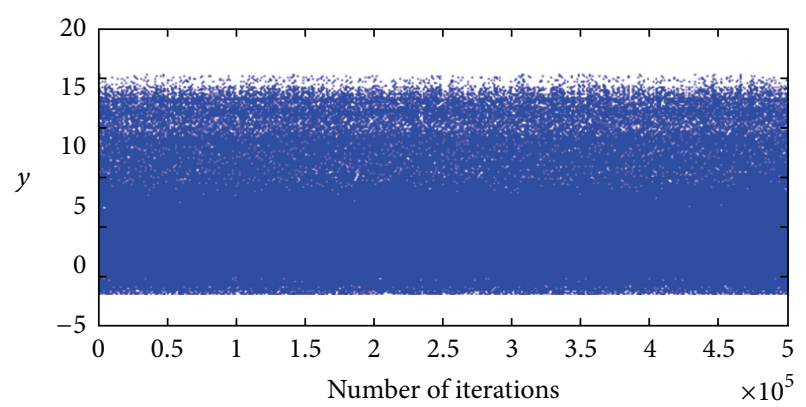

(b)

FIGURE 5: $x_{n}(\mathrm{a}), y_{n}(\mathrm{~b})$ values distribution over iterations.

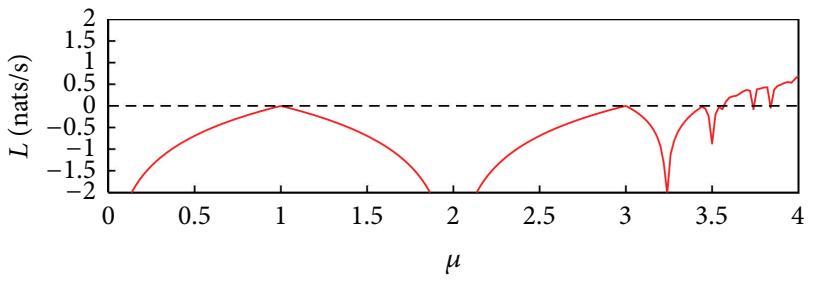

FIGURE 6: Lyapunov spectrum of logistic map.

Step 2 (XOR encryption). Via the algorithm described by (8) below, the XOR passwords sequences $M X, M Y$ are constructed with the chaotic key stream obtained from Step 1. Consider

$$
\begin{aligned}
& m x(n)=\bmod \left(10^{13+\mathrm{key}_{1}^{-1}} \cdot x(n), 256\right), \\
& m y(n)=\bmod \left(10^{14+\mathrm{key}_{1}^{-1}} \cdot y(n), 256\right),
\end{aligned}
$$

$\bmod (. ., 256)$ for making the XOR passwords of an approximate uniform distribution since there are 256 gray levels of each pixel.

After that, to further encrypt the pixel, we XOR this pseudorandom passwords sequences with the gray level in plain image bit by bit though Algorithm 1 .

After that, with the switching method employed, the preciphered image $C^{\prime}$ is obtained, which is able to avoid detection of the chaotic trajectories.

Step 3 (chaotic scrambling). Firstly, remove part of the backward elements in key stream $X$ and $Y$ to make the length of $X^{\prime}$ and $Y^{\prime}$ equal to $M$ and $N$. Map $X^{\prime}(i) X^{\prime}(j)$ with $C_{i, j \operatorname{dim}}^{\prime} 1 \leq \operatorname{dim} \leq 3$ and save the mapping relationship.

Secondly, range $X^{\prime}$ and $Y^{\prime}$ from the smallest to the largest. Scramble $C^{\prime}$ with the mapping relation and then the final ciphered image $C$ is developed.

Apparently, after dual-perturbing effect of Steps 2 and 3 is much better to resist the influence caused by computer precision and can lengthen the period.

3.3. Hiding Scheme and Packet Splitting Scheme. In former process, the plain image is encrypted into the ciphered image. Despite being secure enough, the ciphered image is too notable for attacker due to its strikingly chaotic attribute. In order to make the ciphered image less conspicuous and to increase the robustness and efficiency in real communication, it is essential to use the ciphered image as a watermark which is embedded into several carrier images. The detailed explanation is as follows.

To simplify, the following embedding algorithm is only built with the situation with two carrier images offered. Some feasible adjustments are also declared for the case of more carrier images offered.

To maximize the channel utilization rate for two carrier images' case, the spatial domain based Least Significant Bit (LSB) embedding algorithm will be adopted, because it only needs the carrier image as the same size as the plain image and will not increase the size of the embedded image. For case of more carrier images, transform domain based embedding algorithms are also available and will be much easier to neglect by human perceptual system [33]. However, as a consequence of the limited space, only the LSB algorithm is introduced in detail. Here is the algorithm in steps.

Step 1. Given two color carrier images Ca with size of $\widetilde{M} \times \widetilde{N} \times$ 3 and then scale them to the same size of the plain image $M \times$ $N \times 3$. $\mathrm{Ca}_{i, j \text {,dim }}$ represents pixel in $i$ th row $j$ th column in dimth color space after adjustment. For case of more carrier images, the adjustment is not necessary.

Step 2. For a uint8 format color image, each pixel has 256 grey levels in each RGB dimension, which can be represented by 8 bits binary codes for each dimension. So each pixel in each dimension can be divided into 8 bit planes, which can be represented by a binary image. Because human vision is not sensitive to the least bit plane. So for having two carrier images, the least 4 bits for each of two carrier images are available for hiding the ciphered image. Thus the least 4 bits of each of two carrier images are set to zero from operations, as shown in Algorithm 2.

For case of more carrier images, we can even use part of the least important bit in each carrier image to make the watermark become much more difficult to notice through LSB algorithm.

Step 3. Embed the $32 \mathrm{MD} 5$ parts MD5(i) of plain images into the embedded image with the user set key key $y_{0}$ for watermark detection, decryption, and validation; see Algorithm 3. 


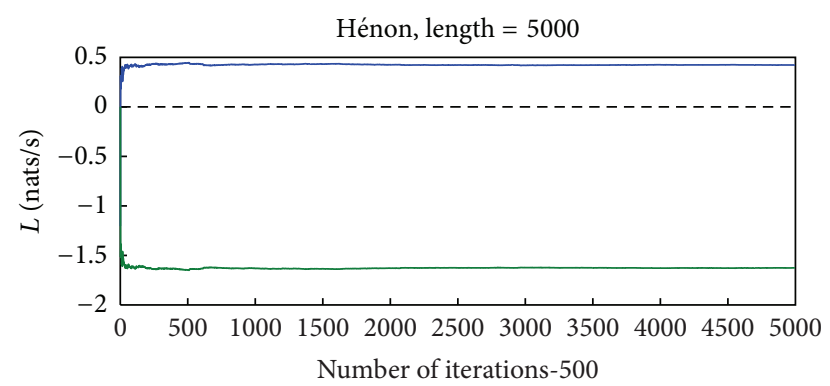

(a)

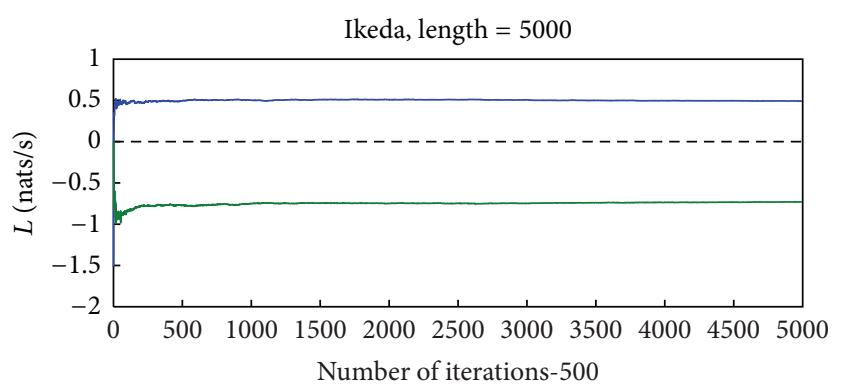

(b)

FiguRE 7: Lyapunov spectrum of Hénon map (a) and Ikeda map (b) (the first 500 iterations are ignored to avoid the influence of initial state).

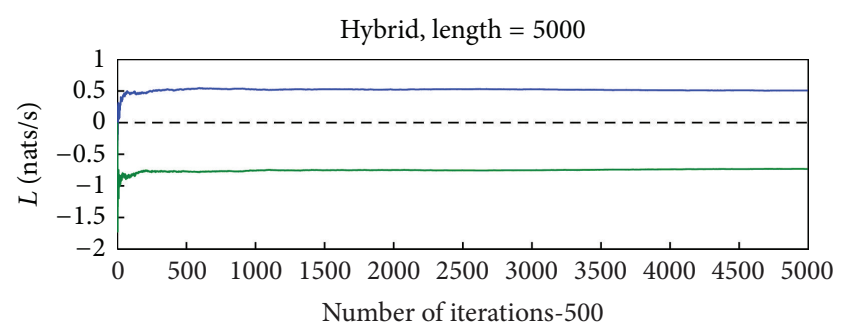

FIGURE 8: Lyapunov spectrum of the new hybrid chaotic map (the first 500 iterations are ignored to avoid the influence of initial state).

Consider

$$
\begin{aligned}
& m_{\mathrm{MD} 5}=\left\lfloor(M-16) \cdot \frac{\mathrm{key}_{0}+1}{10^{\text {length }\left(\mathrm{key}_{0}\right)}+1}\right\rfloor, \\
& n_{\mathrm{MD} 5}=\left\lfloor(N-16) \cdot \frac{\mathrm{key}_{0}+1}{\left.10^{\text {length(key }}\right)+1}\right\rfloor .
\end{aligned}
$$

Step 4. To detect the order of carrier image, a gray pixel should be selected, which does not cover the MD5 value and add the order value into its high bit part for both the transmitter and receiver. This is not necessary for case of two carrier images, but it is necessary for case of more carrier images.

Step 5. Split the ciphered images into two parts. To simplify, the two part is divided into the high 4-bit part and least 4-bit part. Then move the 4 bits with information to the least 4 bits and set the high 4 bits to zero, as shown in Algorithm 4 .

For case of having more carrier images, the remaining chaotic maps can be used for random pixel selection in the ciphered image so as to embed the watermark in the carrier images' locations. This method will further increase the robustness of the watermark and further enhance the security.

Step 6. Embed the two parts of ciphered images into the carrier images from operations, as shown in Algorithm 5.

The switching operation is aimed at well-distributing the two parts into each carrier image and increasing the robustness. For case of more carrier images, the robustness will greatly increase because each embedded image carries less information. With transform domain based embedding algorithms for more carrier images, the robustness can also be increased rapidly. Meanwhile, with channel coding technique, the broken mark can even be recovered.

3.4. Watermark Extraction and Decryption Scheme. The extraction and decryption are the inverse process of the embedding and encryption. Here is the watermark extraction and decryption scheme in five brief steps.

Step 1. Extract the order value and sort the embedded images in right order.

Step 2. Extract the MD5 value of plain image from the embedded image.

Step 3. Extract ciphered image information from several embedded image and join together to recover the ciphered image.

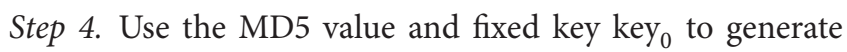
chaotic sequence to realign and decrypt the ciphered image.

Step 5. Calculate the MD5 value of decrypted image and validate with comparison whether the watermark is modified.

\section{Experimental Results}

The scheme is run on MATLAB 8.0 in a personal computer. The $550 \times 366$ color image (Figure 10(a)) sized $590 \mathrm{~KB}$ is used as the plain image. To simplify, two carrier images are chosen as the same $550 \times 366$ color image (Figure 10(c)) sized $590 \mathrm{~KB}$. The secret keys and parameters are set as follows:

$$
\begin{gathered}
\text { key }_{0}=100, \quad w_{0}=s_{0}=0.1, \quad u=0.9, \\
a=1.3, \quad b=4, \quad c=0.4 .
\end{gathered}
$$

The order values are chosen as binary code "0000," "1111" to make a distinction of order between embedded images 1 and 2 in space of $M 1(1,1,1)$ and $M 2(1,1,1)$.

The ciphered image is shown in Figure 10(b). Figure 10(d) illustrates one of embedded images.

The watermark extraction and decryption results are presented in Figure 10. 


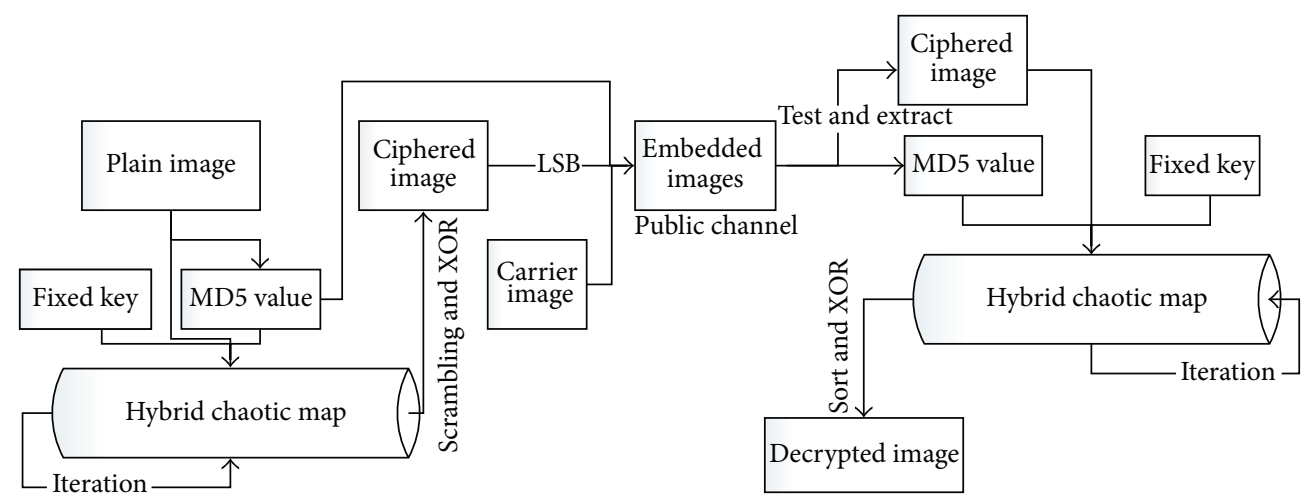

FIGURE 9: Architecture of chaos-based image processing scheme.

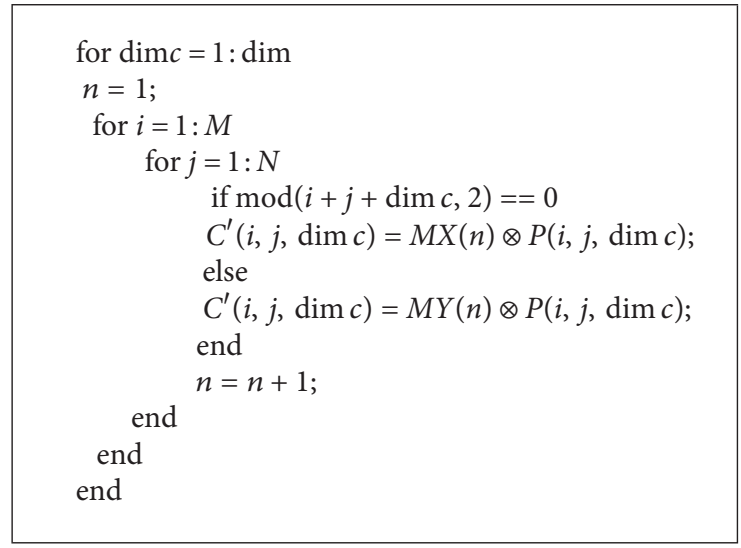

Algorithm 1

In Figure 11(a), we recover plain image when we use the correct key. And Figure 11(b) shows that uses the correct control parameter except $\mathrm{key}_{0}=100+10^{-16}$. The same phenomenon like Figure 11(b) also appears when any control parameters change over $10^{-13}$ and the extraction order is incorrect.

Hence, it can be summarized that even a very tiny difference in keys, control parameters, and the extraction order can cause complete decryption failure. And Figure 12(b) shows the differences between two ciphers, whose corresponding plain images have only one gray pixel different. From the results, it can be concluded that the proposed scheme has high sensitivity to keys and plain image.

\section{Security and Performance Analysis}

Several tests are performed to check the security and robustness of the proposed cryptosystem. Statistical tests are performed, including key space, histogram analysis, information entropy, correlation coefficient, mean square error (MSE), and peak signal-to-noise ratio (PSNR). Likewise, security tests and analysis, such as chosen-plaintext attack and differential attack, are also presented on the scheme. The results are revealed below.
5.1. Key Space. For our proposed cryptosystem, the following keys are included in the key space:

(1) seven control parameters of the hybrid chaotic map; (2) initial fixed key; (3) MD5 values of plain image; (4) two order values; (5) iteration times. To ensure speed, assume the iteration times to be limited less than $10^{3}$ times. Because of the computer precision problem, chaotic state value cannot be infinite. Based on our test results, the chaos behavior works well when accuracy is under $10^{-16}$ for initial fixed key and at least $10^{-13}$ for control parameters. Accordingly, the total key space is $10^{3} \times 10^{13 \times 7} \times 10^{16} \times 10^{6} \times 2^{4 \times 2} \times 2^{128} \gg 2^{128}$, which is sufficiently large to brute-force attack.

5.2. Histogram Analysis. As is known to all, well-behaved encryption algorithms should resist all attacks based on statistical analysis, which requires that the distribution of ciphered image's elements should be close to uniform distribution under any circumstances of plain image's element distribution. Figure 13 is the gray level histogram of both the plain and the ciphered image. From the histogram, it is clear to see that the gray level distribution in ciphered image tends to be uniform distribution, which covers the statistical characteristic of the plain image. 


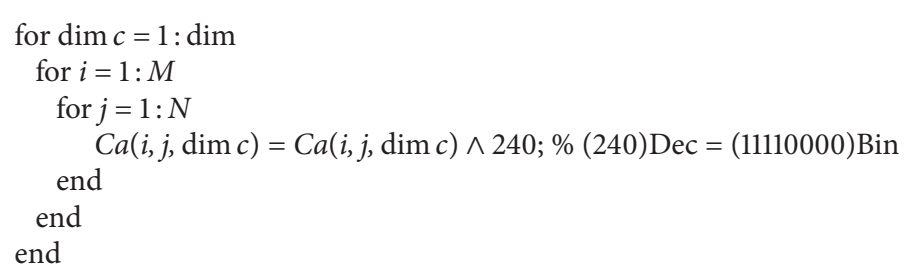

Algorithm 2

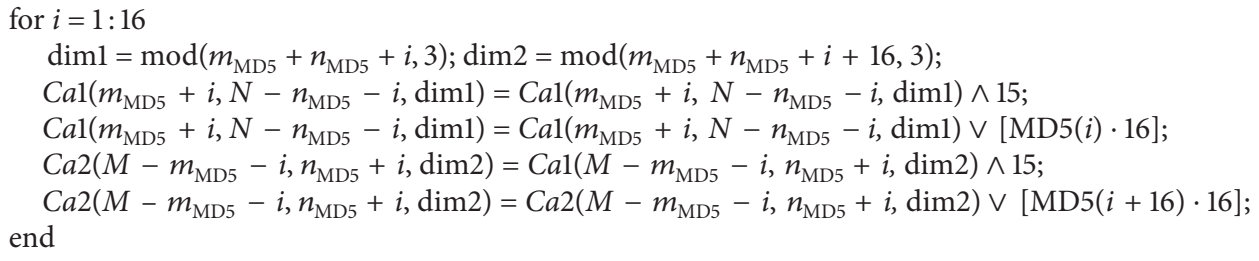

Algorithm 3

5.3. Information Entropy. According to the Shannon theory [34], information entropy is the most essential expressive feature of randomness. We can calculate information entropy $H(m)$ over (11):

$$
H(m)=-\sum_{i} p\left(m_{i}\right) \log _{2} \frac{1}{p\left(m_{i}\right)},
$$

where $m$ is the message, $m_{i}$ represents the probability of symbol $m_{i}$, and the entropy is expressed in bits. So to a pixel of which a symbol is encoded by $8 \times 3=24$ bits ( 3 means 3 color planes), when ideally random, entropy should be 24 ; in general, the entropy value of the source is smaller than 24 but ought to be close to be ideal. From our calculation, the entropy obtained from the ciphered image equals to 23.9973, which is very close to the ideal value 24 , while the entropy obtained from the plain image is only 21.8747 .

5.4. Correlation Coefficient. Correlation coefficient reflects the connection between pixels. According to the following formula, we obtain the correlation coefficients of two adjacent pixels and of corresponding pixels between two different images

$$
r_{x y}=\frac{\operatorname{cov}(x, y)}{\sqrt{D(x)} \sqrt{D(y)}},
$$

where $\operatorname{cov}(x, y)=(1 / N) \sum_{i=1}^{N}\left(x_{i}-E(x)\right)\left(y_{i}-E(y)\right), E(x)=$ $(1 / N) \sum_{i=1}^{N} x_{i}, D(x)=(1 / N) \sum_{i=1}^{N}\left(x_{i}-E(x)\right)^{2}$.

Table 2 displays the results of correlation coefficients, and Figure 14 shows the correlation distribution. Results point out that the correlation between two adjacent pixels in ciphered image and between corresponding pixels in two different images is tremendously small, which displays its great encryption performance.
5.5. Noise Interference and Mean Square Error (MSE). Mean square error (MSE) is key value to quantify the difference between two images in statistics, which can demonstrate the average difference between different ciphered images and between plain and ciphered images. It also reflects the robustness in watermarks when the channel is not ideal. The definition of mean square error is according to the following formula:

$$
\operatorname{MSE}=\frac{1}{M \cdot N \cdot \operatorname{dim}} \sum\left(P_{i, j, \operatorname{dim}}-\widehat{D}_{i, j, \operatorname{dim}}\right)^{2},
$$

where $\widehat{D}_{i, j \text {,dim }}$ means another image's corresponding pixel.

Table 3 shows the results of MSE. Results indicate that the difference between different ciphered images and between plain and ciphered image is hugly immense, while the difference between decrypted and plain images both with and without the noise interference is relatively small, which is illustrated in Figure 15 and shows both the great encryption performance and the great robustness of the scheme.

5.6. Peak Signal-to-Noise Ratio (PSNR). Peak signal-to-noise ratio (PSNR) is a ratio between the maximum possible power of a signal and the power of corrupting noise that affects the fidelity of its representation. Because many signals have a very wide dynamic range, PSNR is usually expressed in terms of the logarithmic decibel scale [35]. The PSNR is defined as

$$
\mathrm{PSNR}=10 \log _{10}\left(\frac{\mathrm{MAX}_{I}^{2}}{\mathrm{MSE}}\right) .
$$

Here, MAX $_{I}$ is the maximum possible pixel value of the image. When the pixels are represented using 8 bits per sample, $\operatorname{MAX}_{I}=255$. Typical values for the PSNR in image and video compression are between 30 and $50 \mathrm{~dB}$, where higher is better [35]. For previous simulation of noise interference, PSNR of the decrypted image with 0.001 Gaussian 


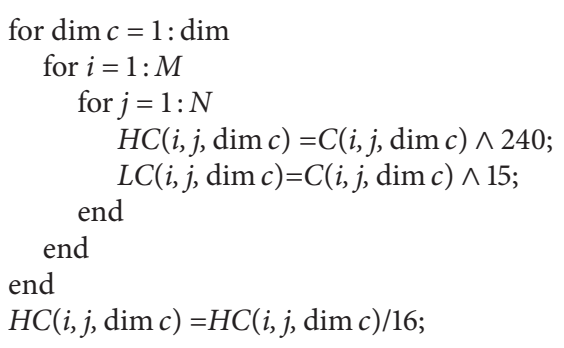

Algorithm 4

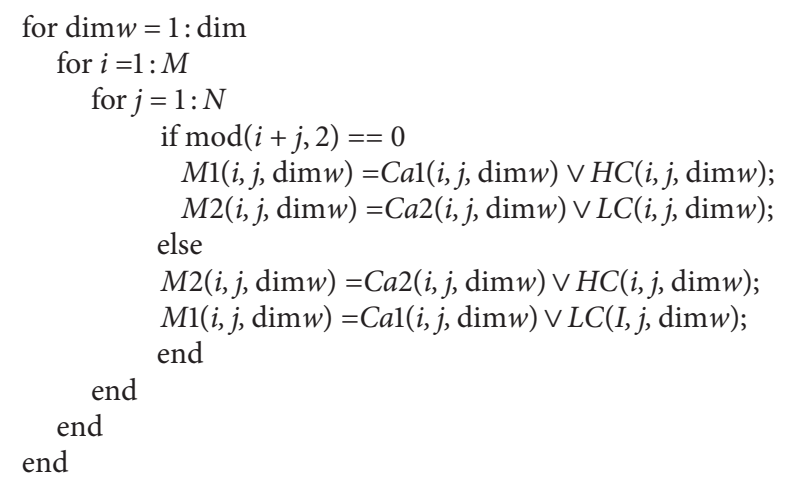

Algorithm 5

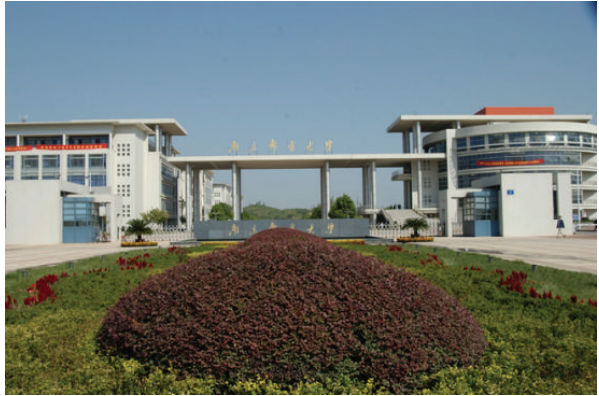

(a) Plain image

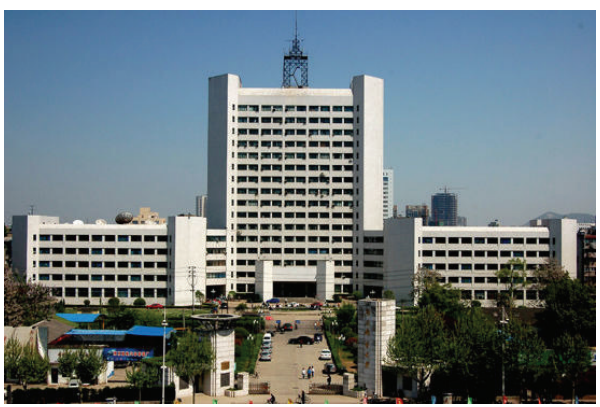

(c) Carrier image

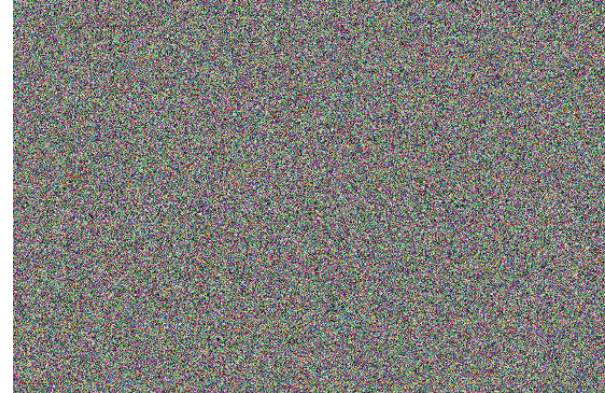

(b) Cipher image

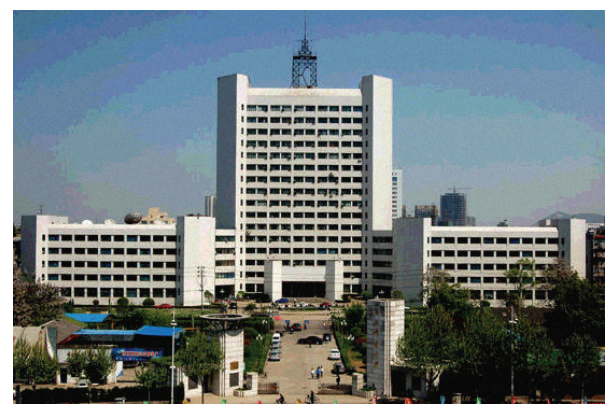

(d) Watermark embedded

FIGURE 10: Encryption and watermark embedding results. 


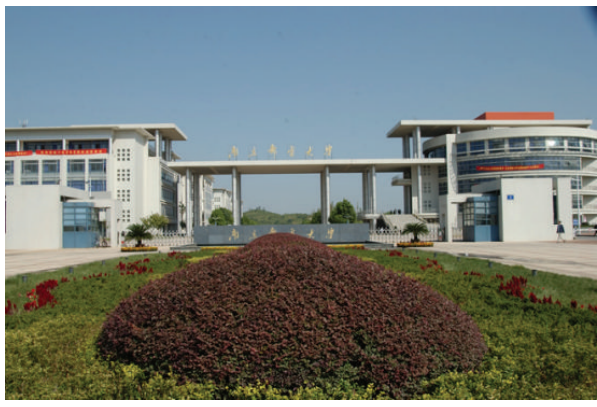

(a) Success of decryption

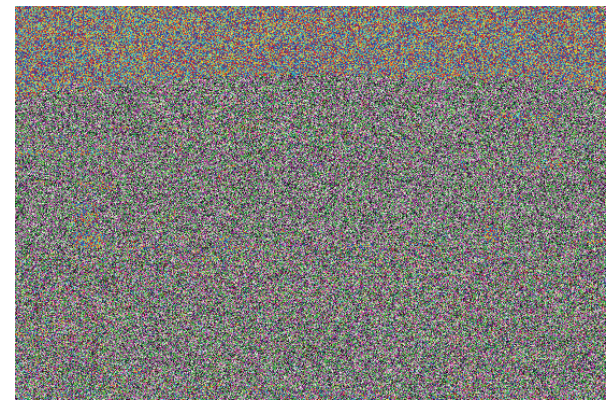

(b) Failure of decryption

FIGURE 11: Watermark extraction and decryption results.

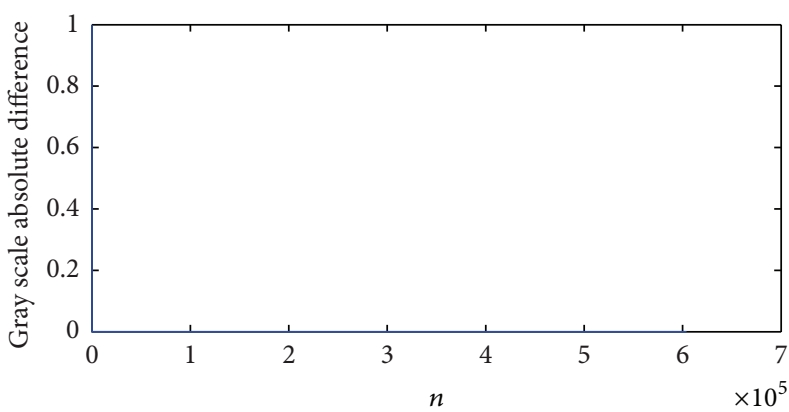

(a) Differences between only one pixel different plain images

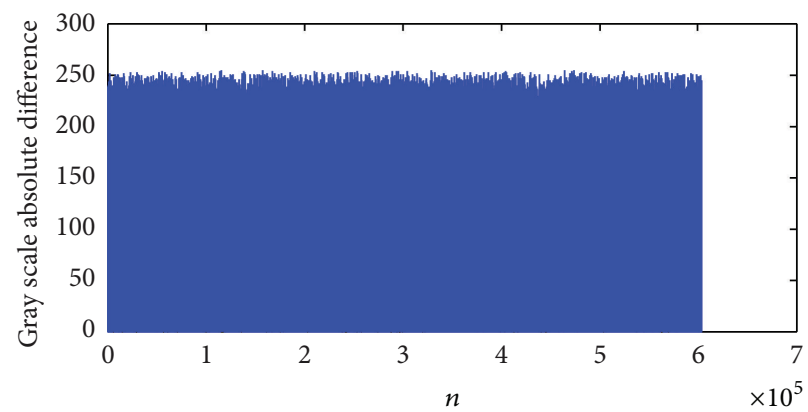

(b) Differences between its corresponding ciphers

Figure 12: Differences comparison.
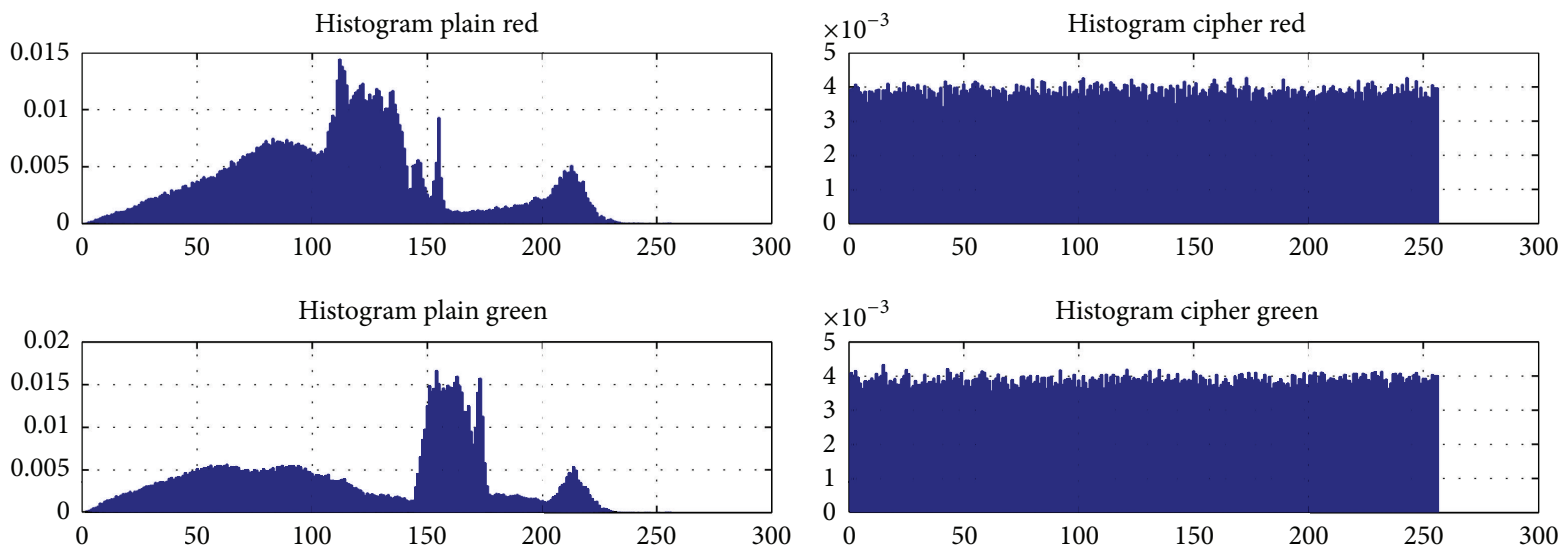

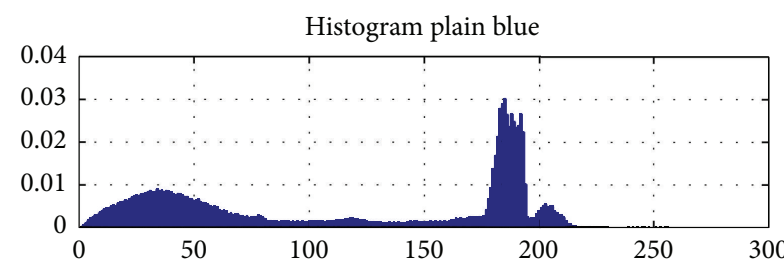

(a) Gray level histogram of plain image

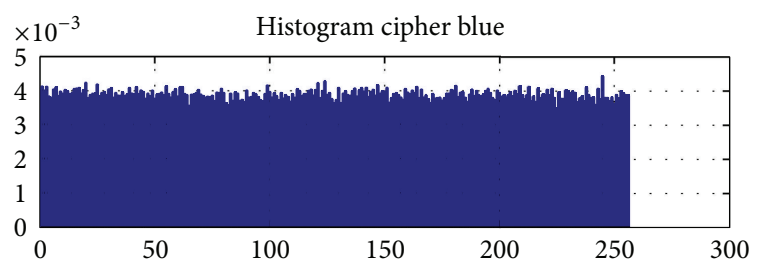

(b) Gray level histogram of its corresponding ciphered image

FIGURE 13: Gray level histogram comparisons. 


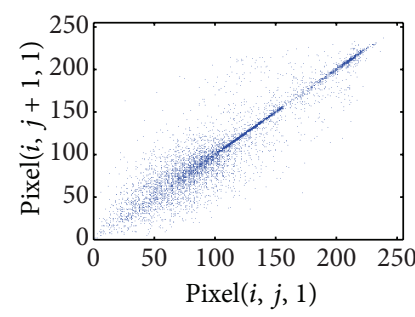

(a) Vertical correlation of plain image on red plane

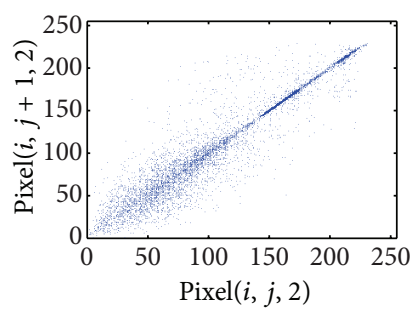

(e) Vertical correlation of plain image on green plane

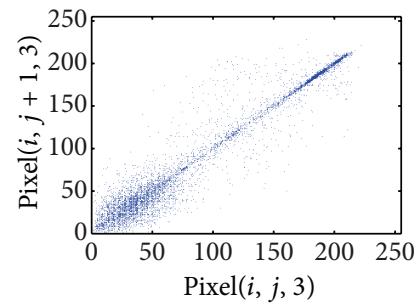

(i) Vertical correlation of plain image on blue plane

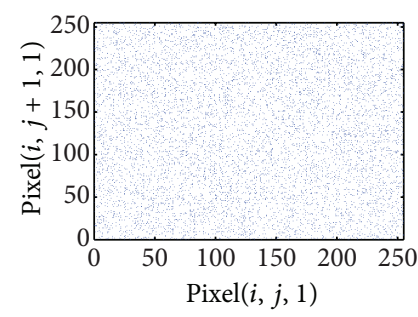

(b) Vertical correlation of ciphered image on red plane

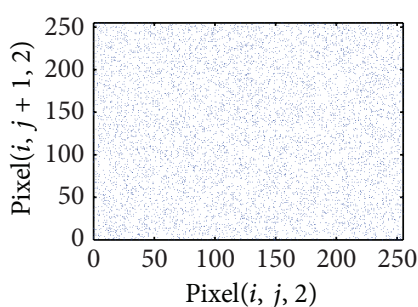

(f) Vertical correlation of ciphered image on green plane

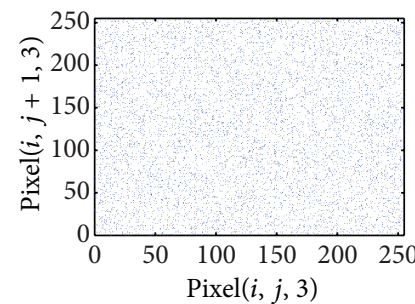

(j) Vertical correlation of ciphered image on blue plane

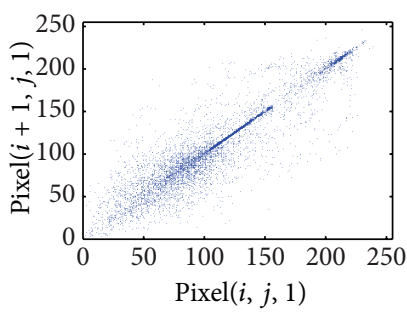

(c) Horizontal correlation of plain image on red plane

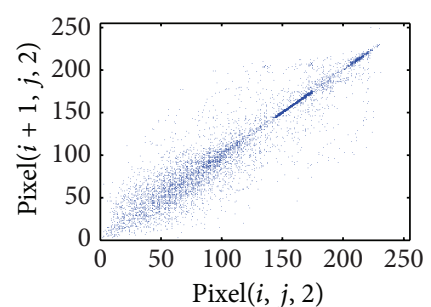

(g) Horizontal correlation of plain image on green plane

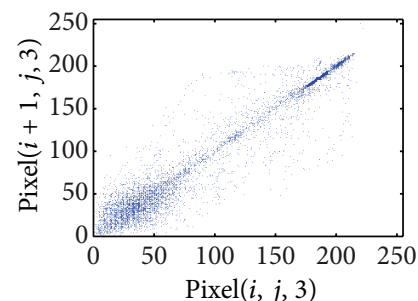

(k) Horizontal correlation of plain image on blue plane

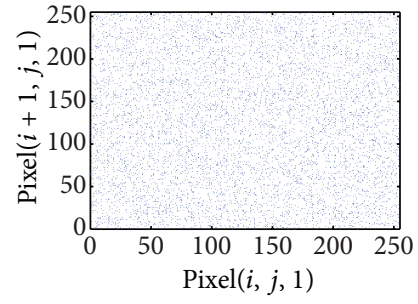

(d) Horizontal correlation of ciphered image on red plane

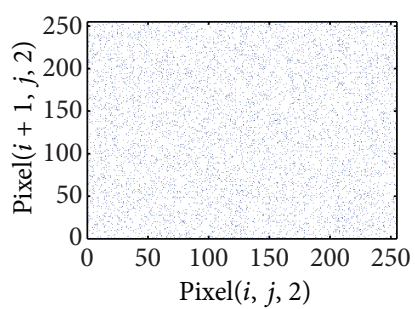

(h) Horizontal correlation of ciphered image on green plane

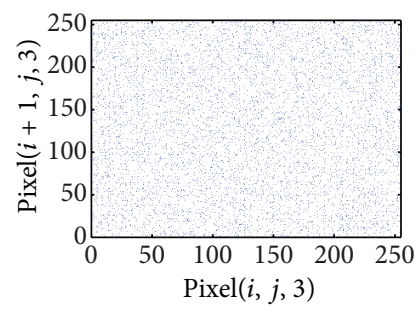

(l) Horizontal correlation of ciphered image on blue plane

FIGURE 14: Vertical (left) and horizontal (right) correlation of plain image and ciphered image.

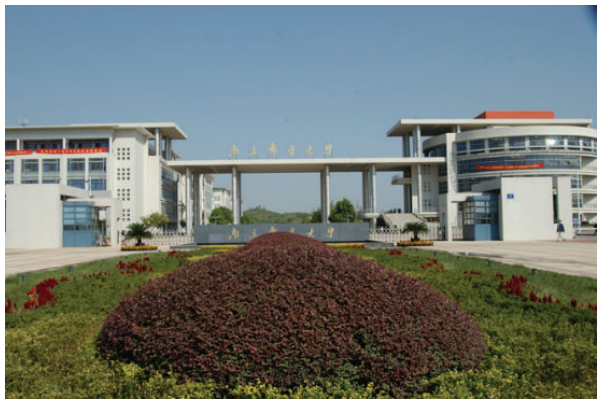

(a) Decrypted image without noise

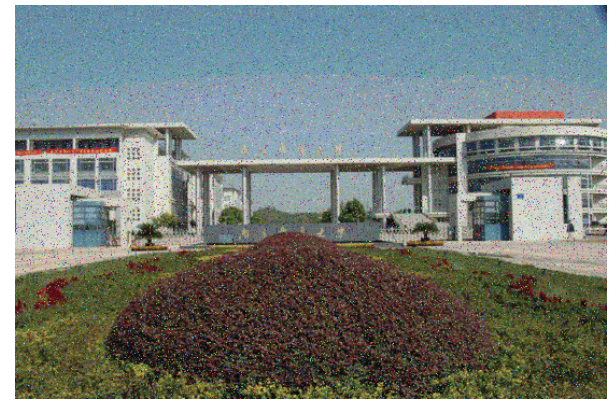

(b) Decrypted image with 0.01 Gaussian noise

FIGURE 15: Noise interference comparisons.

TABLE 2: Correlation coefficient comparison.

\begin{tabular}{cccccc}
\hline & & \multicolumn{2}{c}{ Between two adjacent pixels } & \multicolumn{2}{c}{ Between corresponding pixels in two different images } \\
& & Plain image & Ciphered image & Different ciphered images & Plain-ciphered $^{2}$ \\
\hline \multirow{3}{*}{ Correlation coefficient } & Vertical & 0.96684049 & $7.16211445 \times 10^{-5}$ & & \\
& Horizontal & 0.954339329 & -0.0010516 & $7.29256967 \times 10^{-4}$ & -0.00135729535 \\
& Diagonal & 0.935083629 & 0.00151382 & & \\
\hline
\end{tabular}

\footnotetext{
${ }^{2}$ The two difference ciphered images' corresponding plain images only have one gray pixel in difference.
} 
TABLE 3: MSE comparison.

\begin{tabular}{lcc}
\hline MSE & $\begin{array}{c}\text { Corresponding } \\
\text { ciphered image }\end{array}$ & $\begin{array}{c}\text { Corresponding decrypted } \\
\text { image }\end{array}$ \\
\hline Plain image & 127.7904 & $\begin{array}{c}0 \text { (channel without any noise) } \\
36.9984 \text { (channel with } 0.001 \\
\text { Gaussian noise) }\end{array}$ \\
$\begin{array}{l}\text { Different } \\
\text { ciphered image }\end{array}$ & 117.2472 & 106.0239 \\
\hline
\end{tabular}

noise interference is $32.4490 \mathrm{~dB}$, which denotes the decent robustness of the system.

5.7. Plaintext-Chosen Attacks Analysis. Many encryption algorithms are faced with the risk of being broken by the chosen-plaintext analysis. But the proposed scheme in this paper combining the chaotic map's initial key with the plain image's MD5 value, which is one-way dependent on the plain image's information, makes the chosen-plaintext attack unavailable. Because even though much progress has been made in the field of attacking the MD5 algorithm in recent years [36], the price of finding a group of pictures with the same MD5 value and the great characteristic for attacking analysis, such as plaintext-chosen attack and differential attack, is still extremely expensive. Meanwhile, by using the block cipher of MD5 like the 32 blocks in Section 3.2.1, the difficulty of MD5 collide is increased. As a result, just like the simulation results in Section 4, for different plain images (even a tiny little change), the corresponding sequences are totally different on account of the property of MD5 hash and chaos. By these means, our proposed algorithm is robust to plaintext-chosen attack.

5.8. Differential Attacks Analysis. There are two most common magnitudes-Number of Pixels Change Rate (NPCR) and Unified Average Changing Intensity (UACI), which are used to evaluate the strength of image encryption in regard to differential attacks. Conventionally, a high NPCR and UACI score usually signifies a high resistance to differential attacks. The definitions of NPCR and UACI are stated by (15) [37]. To do the test, let us assume two ciphered images $\left(C, C^{\prime}\right)$ whose corresponding plain images only have one pixel difference initially:

$$
\begin{aligned}
\mathrm{NPCR} & =\frac{\sum_{i, j, \operatorname{dim}} D(i, j, \operatorname{dim})}{W \times H \times \operatorname{dim}} \times 100 \%, \\
\mathrm{UACI}= & \frac{1}{W \times H \times \operatorname{dim}} \\
& \times\left[\sum_{i, j, \mathrm{dim}} \frac{\left|C(i, j, \operatorname{dim})-C^{\prime}(i, j, \operatorname{dim})\right|}{255}\right] \\
& \times 100 \%,
\end{aligned}
$$

where $W$ and $H$ represent the width and height of the image, respectively. $C(i, j, \operatorname{dim})$ and $C^{\prime}(i, j, \operatorname{dim})$ are the ciphered images before and after one gray level of one pixel in the plain image is changed. For position $(i, j, \operatorname{dim})$, if $C(i, j, \operatorname{dim}) \neq C^{\prime}(i, j, \operatorname{dim})$, then $D(i, j, \operatorname{dim})=1$; else $D(i, j$, $\operatorname{dim})=0$.

Based on our test results, the NPCR is $99.67 \%$ and the UACI is $33.45 \%$, which proves that the proposed algorithm is very sensitive to small changes in the plain image and can survive to differential attack.

\section{Conclusion}

In this paper, an innovative hybrid chaotic mapping system has been crafted through compositing with three classic chaotic maps. Additionally, its application on image encryption and hiding has also been discussed with a demonstration scheme. Notably, from our test results, the new map has the largest positive Lyapunov exponent and the second largest negative Lyapunov exponent over the original 2D chaotic maps. What is more, this constructing method is of profound theoretical and practical prominence because with the plan of composition, more new and sensitive chaotic maps will come to the fore much easily, which could extremely avoid the threat of known-system based attack. In the image encryption scheme, the new hybrid chaotic map has been functioned as the key stream generator for encryption in view of its impressive efficiency and pseudorandom properties. On top of that, to improve the facilities of resisting plaintext-chosen and differential attacks and to make pseudorandom keys much more unpredictable, the MD5 hash of plain image has been used as part of initial condition and control parameter to perturb the trajectory during iterations. Simulation results and security analysis have disclosed that the proposed scheme has marvelous efficiency and robustness and is proved to be theoretically unbreakable by conventional attacks.

\section{References}

[1] N. Doraswamy and D. Harkins, Ipsec: the New Security Standard For the Internet, Intranet and Virtual Private Network, Prentice Hall, New York, NY, USA, 2003.

[2] B. Schneier, Applied Cryptography: Protocols, Algorithms, and Source Code in C, John Wiley \& Sons, New York, NY, USA, 2007.

[3] J. K. Su, F. Hartung, and B. Girod, "Digital watermarking of text, image, and video documents," Computers and Graphics, vol. 22, no. 6, pp. 687-695, 1998.

[4] M. Hénon, "A two-dimensional mapping with a strange attractor," Communications in Mathematical Physics, vol. 50, no. 1, pp. 69-77, 1976.

[5] A. Uhl and A. Pommer, Image and Video Encryption: From Digital Rights Management to Secured Personal Communication, vol. 15, Springer, New York, NY, USA, 2004.

[6] L. Kocarev and S. Lian, Chaos-Based Cryptography: Theory, Algorithms and Applications, vol. 354, Springer, New York, NY, USA, 2011.

[7] P. Stavroulakis and M. Stamp, Handbook of Information and Communication Security, Springer, New York, NY, USA, 2010.

[8] M. E. Smid and D. K. Branstad, "Data encryption standard: past and future," Proceedings of the IEEE, vol. 76, pp. 550-559, 1988.

[9] S. William, Cryptography and Network Security, 4/E, Pearson Education, New Delhi, India, 2006. 
[10] J. L. Freitas and R. R. Oliveira, "Implementação, comparação e análise dos algoritmos IDEA e DES," in I Encontro Regional em Modelagem e Análise Computacional de Sistemas (ERMACS '04), Goiânia, Brazil, 2004.

[11] J. Daemen and V. Rijmen, The Design of Rijndael: AES-the Advanced Encryption Standard, Springer, New York, NY, USA, 2002.

[12] R. Matthews, "On the derivation of a "chaotic" encryption algorithm," Cryptologia, vol. 13, no. 1, pp. 29-42, 1989.

[13] K.-W. Wong, B. S.-H. Kwok, and W.-S. Law, "A fast image encryption scheme based on chaotic standard map," Physics Letters A, vol. 372, no. 15, pp. 2645-2652, 2008.

[14] C. K. Huang and H. H. Nien, "Multi chaotic systems based pixel shuffle for image encryption," Optics Communications, vol. 282, no. 11, pp. 2123-2127, 2009.

[15] S. Behnia, A. Akhshani, S. Ahadpour, H. Mahmodi, and A. Akhavan, "A fast chaotic encryption scheme based on piecewise nonlinear chaotic maps," Physics Letters A, vol. 366, no. 4-5, pp. 391-396, 2007.

[16] X.-Y. Wang, L. Yang, R. Liu, and A. Kadir, "A chaotic image encryption algorithm based on perceptron model," Nonlinear Dynamics, vol. 62, no. 3, pp. 615-621, 2010.

[17] M. S. Baptista, "Cryptography with chaos," Physics Letters A, vol. 240, no. 1-2, pp. 50-54, 1998.

[18] G. Álvarez, F. Montoya, M. Romera, and G. Pastor, "Cryptanalysis of an ergodic chaotic cipher," Physics Letters A, vol. 311, no. 2-3, pp. 172-179, 2003.

[19] A. Kiani-B, K. Fallahi, N. Pariz, and H. Leung, "A chaotic secure communication scheme using fractional chaotic systems based on an extended fractional Kalman filter," Communications in Nonlinear Science and Numerical Simulation, vol. 14, no. 3, pp. 863-879, 2009.

[20] G. C. Wu and D. Baleanu, "Discrete fractional logistic map and its chaos," Nonlinear Dynamics, 2013.

[21] N. K. Pareek, V. Patidar, and K. K. Sud, "Cryptography using multiple one-dimensional chaotic maps," Communications in Nonlinear Science and Numerical Simulation, vol. 10, no. 7, pp. 715-723, 2005.

[22] N. K. Pareek, V. Patidar, and K. K. Sud, "Image encryption using chaotic logistic map," Image and Vision Computing, vol. 24, no. 9, pp. 926-934, 2006.

[23] G. Álvarez, F. Montoya, M. Romera, and G. Pastor, "Cryptanalysis of a discrete chaotic cryptosystem using external key," Physics Letters A, vol. 319, no. 3-4, pp. 334-339, 2003.

[24] C. Li, S. Li, M. Asim, J. Nunez, G. Alvarez, and G. Chen, "On the security defects of an image encryption scheme," Image and Vision Computing, vol. 27, no. 9, pp. 1371-1381, 2009.

[25] S. Li, X. Mou, Y. Cai, Z. Ji, and J. Zhang, "On the security of a chaotic encryption scheme: problems with computerized chaos in finite computing precision," Computer Physics Communications, vol. 153, no. 1, pp. 52-58, 2003.

[26] T. Gao and Z. Chen, "A new image encryption algorithm based on hyper-chaos," Physics Letters A, vol. 372, no. 4, pp. 394-400, 2008.

[27] H. Liu and X. Wang, "Color image encryption based on onetime keys and robust chaotic maps," Computers \& Mathematics with Applications, vol. 59, no. 10, pp. 3320-3327, 2010.

[28] P. F. Verhulst, "Recherches mathématiques sur la loi d'accroissement de la population," Nouveaux Mémoires de l'académie Royale des Sciences et Belles-Lettres de Bruxelles, vol. 18, pp. 3-38, 1845.
[29] R. M. May, "Biological populations with nonoverlapping generations: stable points, stable cycles, and chaos," Science, vol. 186, no. 4164, pp. 645-647, 1974.

[30] K. Ikeda, "Multiple-valued stationary state and its instability of the transmitted light by a ring cavity system," Optics Communications, vol. 30, no. 2, pp. 257-261, 1979.

[31] A. Wolf, J. B. Swift, H. L. Swinney, and J. A. Vastano, "Determining Lyapunov exponents from a time series," Physica D, vol. 16, no. 3, pp. 285-317, 1985.

[32] G. Elert, The Chaos Hypertextbook: Measuring Chaos, vol. 10, 2003.

[33] K. Mahmoud, S. Datta, and J. Flint, Frequency Domain Watermarking: An Overview, 2003.

[34] C. E. Shannon, "A mathematical theory of communication," ACM SIGMOBILE Mobile Computing and Communications Review, vol. 5, pp. 3-55, 2001.

[35] Q. Huynh-Thu and M. Ghanbari, "Scope of validity of PSNR in image/video quality assessment," Electronics Letters, vol. 44, no. 13, pp. 800-801, 2008.

[36] A. Cilardo, L. Coppolino, N. Mazzocca, and L. Romano, "Elliptic curve cryptography engineering," Proceedings of the IEEE, vol. 94, no. 2, pp. 395-405, 2006.

[37] Y. Wu, J. P. Noonan, and S. Agaian, "NPCR and UACI randomness tests for image encryption," Cyber Journals: Multidisciplinary Journals in Science and Technology, Journal of Selected Areas in Telecommunications, pp. 31-38, 2011. 


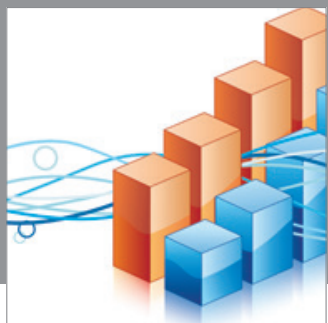

Advances in

Operations Research

mansans

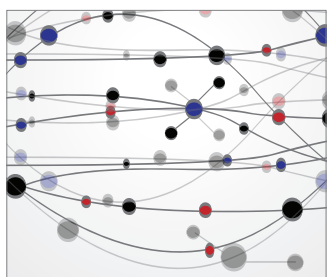

The Scientific World Journal
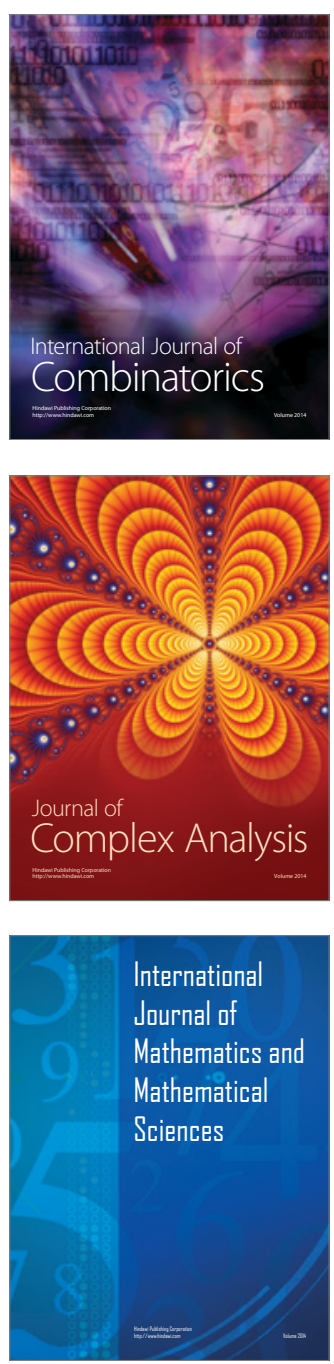
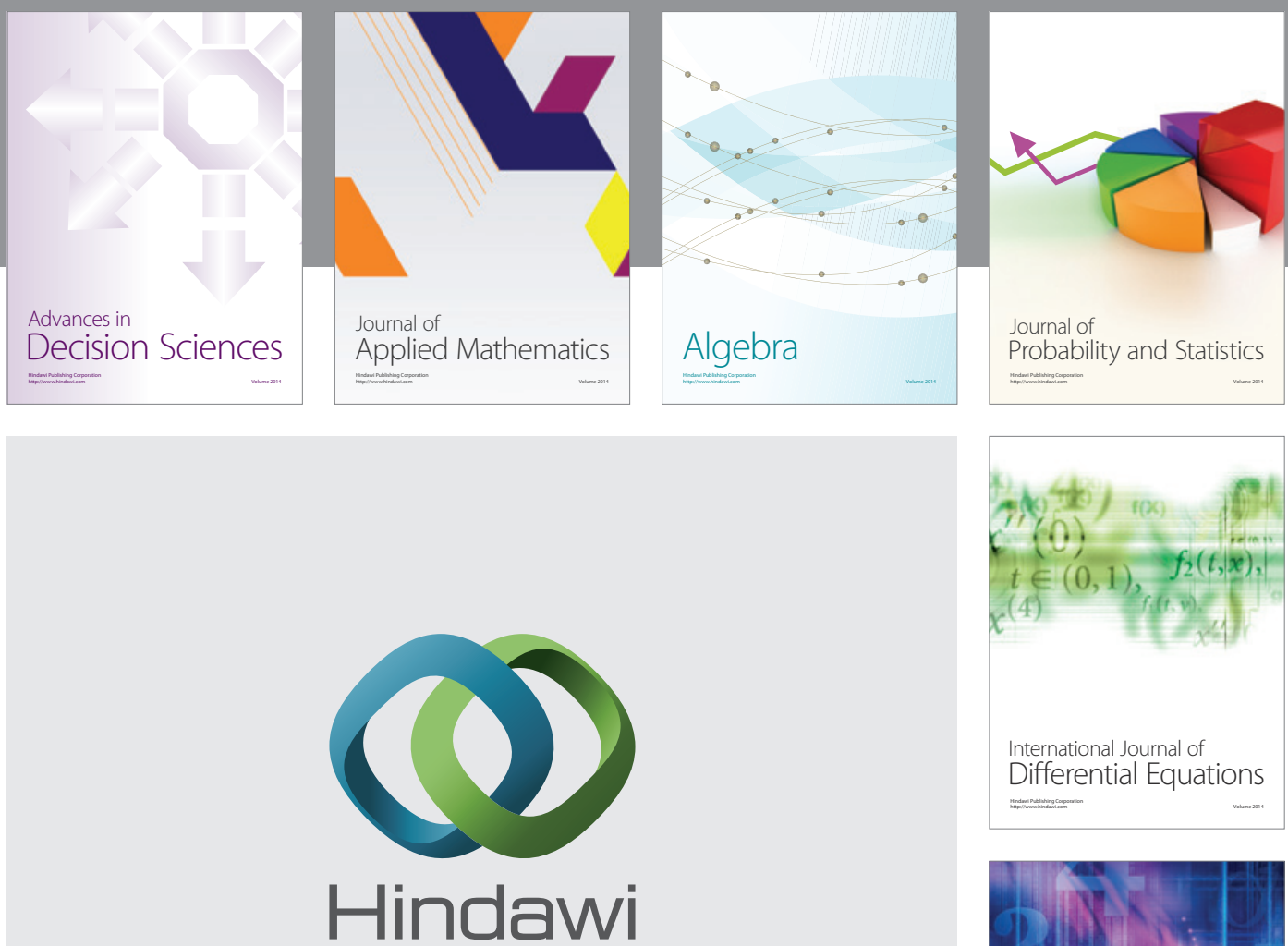

Submit your manuscripts at http://www.hindawi.com
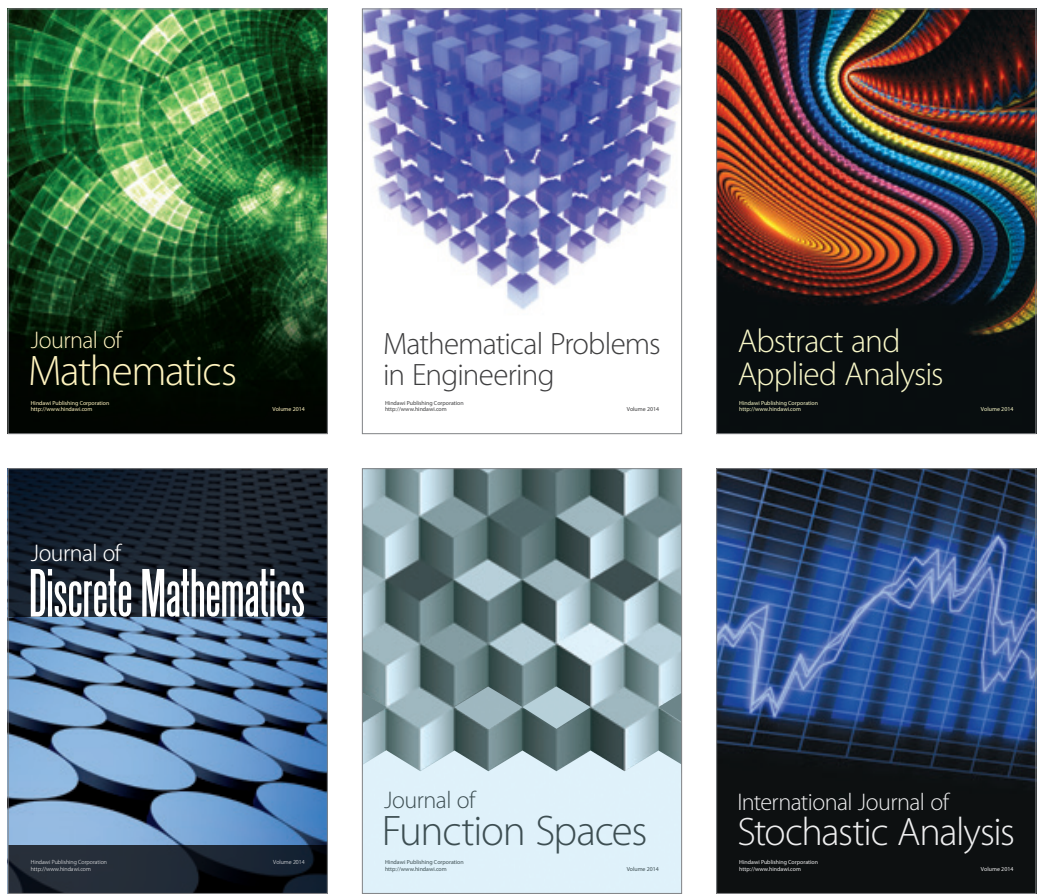

Journal of

Function Spaces

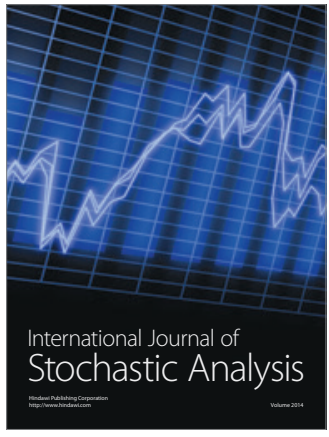

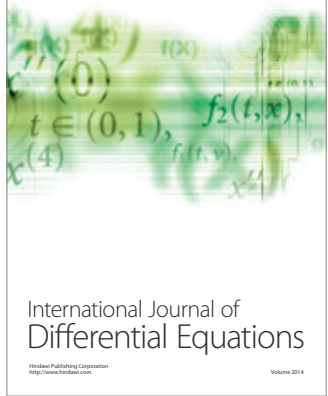
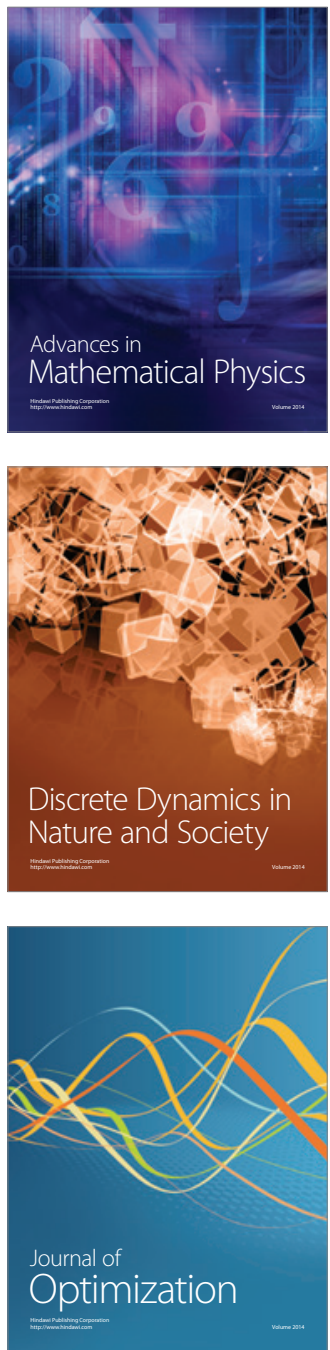